\title{
Preparation of Gd Complex-Immobilized Silica Particles and Their Application to MRI
}

\author{
Yoshio Kobayashi, ${ }^{1}$ Hikaru Morimoto, ${ }^{1}$ Tomohiko Nakagawa, ${ }^{2}$ Yohsuke Kubota, ${ }^{2}$ \\ Kohsuke Gonda, ${ }^{2}$ and Noriaki Ohuchi ${ }^{2}$ \\ ${ }^{1}$ Department of Biomolecular Functional Engineering, College of Engineering, Ibaraki University, 4-12-1 Naka-Narusawa-cho, \\ Hitach-shi, Ibaraki 316-8511, Japan \\ ${ }^{2}$ Division of Surgical Oncology, Graduate School of Medicine, Tohoku University, 2-1 Seiryo-machi, Aoba-ku, Sendai-shi, \\ Miyagi 980-8574, Japan \\ Correspondence should be addressed to Yoshio Kobayashi; ykoba@mx.ibaraki.ac.jp
}

Received 13 February 2013; Accepted 25 March 2013

Academic Editors: Y. A. Koksharov and P. Perriat

Copyright ( 2013 Yoshio Kobayashi et al. This is an open access article distributed under the Creative Commons Attribution License, which permits unrestricted use, distribution, and reproduction in any medium, provided the original work is properly cited.

\begin{abstract}
A preparation method for Gd-ethylenediaminetetraacetic acid disodium salt dihydrate (ETDA) complex-immobilized silica particles $\left(\mathrm{Gd}-\mathrm{EDTA} / \mathrm{SiO}_{2}\right)$ is proposed. Preparation of spherical silica particles was performed by a sol-gel method at $35^{\circ} \mathrm{C}$ using $0.2 \mathrm{M}$ tetraethylorthosilicate, $25 \mathrm{M} \mathrm{H}_{2} \mathrm{O}$, and $0.01 \mathrm{M} \mathrm{NaOH}$ in ethanol, which produced silica particles with an average size of $80.4 \pm 14.9 \mathrm{~nm}$. Immobilization of Gd-EDTA on the silica particles was conducted at $35^{\circ} \mathrm{C}$ by introducing amino groups on the silica particles with (3-aminopropyl)trimethoxysilane at $\mathrm{pH} 3\left(\mathrm{NH}_{2} / \mathrm{SiO}_{2}\right)$ and then making Gd-EDTA act on the $\mathrm{NH}_{2} / \mathrm{SiO}_{2}$ particles at $\mathrm{pH}$ 5. The as-prepared Gd-EDTA/SiO 2 particle colloid solution was concentrated up to a $\mathrm{Gd}$ concentration of $0.347 \mathrm{mM}$ by centrifugation. The sphere structure of $\mathrm{Gd}-\mathrm{EDTA} / \mathrm{SiO}_{2}$ particles was undamaged, and the colloid solution was still colloidally stable, even after the concentrating process. The concentrated Gd-EDTA/SiO 2 colloid solution revealed good MRI properties. A relaxivity value for $\mathrm{T}_{1}$-weighted imaging was as high as $5.15 \mathrm{mM}^{-1} \mathrm{~s}^{-1}$, that was comparable to that for a commercial Gd complex contrast agent.
\end{abstract}

\section{Introduction}

Gadolinium complexes (GdC) function as contrast agents for magnetic resonance imaging (MRI) [1-8], and solutions dissolving the GdC homogeneously at molecular level are available commercially. The GdC molecules flow fast in living bodies. This means that they are quickly discharged from the living bodies, which results in a difficulty in taking highcontrast images for a long period with the use of the solutiontype commercial agents.

Not the GdC solution but colloid solution of GdC immobilized with particles is promising as a new contrast agent that can be expected to stay in living bodies for a long period, because the particles are dragged from fluid to a direction opposite to the fluid due to their projected area larger than molecules, which controls flow of the particles in liquid. Consequently, the immobilization will increase residence time of $\mathrm{GdC}$, which makes it possible to take clear MRI images for a long term. Particles used for the immobilization are required to unaggregate in living bodies, because aggregation of the particles prevents smooth flow of body fluid. Since silica particles prepared by a sol-gel method are colloidally stable [9-11], GdC-immobilized silica particles may function as the MRI contrast agent that shows long residence time and nonaggregation in living bodies.

Lux et al. produced particles composed of Gd oxide core and silica-related compound shell for bioimaging [12]. Our group also proposed a method for fabricating silica-coated Gd compound nanoparticles, which was a combination process of a homogeneous precipitation method and a solgel method [13]. Their particles contain the Gd compound particles as core. Besides the core-shell particles, various methods for immobilizing GdCs on silica surfaces of silica 
particles or silica-coated particles have been proposed by several researchers [14-18]. An alternative method for the GdCs immobilizing is proposed in the present work. First, silica particles fabricated with the sol-gel method were surfacemodified with amino groups by using a silane coupling reagent with amino group $\left(\mathrm{NH}_{2} / \mathrm{SiO}_{2}\right)$. Then, ethylenediaminetetraacetic acid (EDTA), a representative ligand for metal ion, was introduced on the particle surface through a reaction between the amino groups on particle surface and carboxyl groups of the EDTA $\left(\mathrm{EDTA} / \mathrm{SiO}_{2}\right)$. Finally, $\mathrm{Gd}^{3+}$ ions were immobilized on the particle surface through formation of $\mathrm{Gd}$ complexes (Gd-EDTA/SiO ${ }_{2}$ ). The present work also studied on MRI properties of the colloid solution of $\mathrm{Gd}-\mathrm{EDTA} / \mathrm{SiO}_{2}$ particles.

\section{Experimental}

2.1. Chemicals. A starting reagent for producing silica particles was tetraethylorthosilicate (TEOS) (95\%). Solvent and catalyst for the sol-gel reaction of TEOS were ethanol (99.5\%) and sodium hydroxide $(\mathrm{NaOH})$ solution $(5 \mathrm{M})$, respectively. (3-Aminopropyl)trimethoxysilane (APMS) (Sigma-Aldrich, 97\%) was used for surface modification of silica particles. EDTA disodium salt dihydrate (Sigma-Aldrich, 99.0-101.0\%) and dimethylformamide (DMF) (>99.5\%) were used as a ligand for $\mathrm{Gd}^{3+}$ and a solvent of EDTA in introduction of EDTA on particles, respectively. A source of $\mathrm{Gd}^{3+}$ for forming Gd-EDTA complex was gadolinium nitrate hexahydrate $\left(\mathrm{Gd}\left(\mathrm{NO}_{3}\right)_{3} \cdot 6 \mathrm{H}_{2} \mathrm{O}\right)$ (99.5\%). 2,4,6-Trinitrobenzennesulfonic acid sodium salt dehydrate (TNBS) (Wako Pure Chemical, 98\%) was used for confirming presence of amino groups in colloid solutions. Particle colloid solutions produced in the present work were compared to Magnevist (Bayer Co., Ltd., $0.5 \mathrm{M} \mathrm{Gd}$ ), which is one of the representative of MRI contrast agents. All the chemicals except for APMS, EDTA, and TNBS were purchased from Kanto Chemical Co., Inc. and used as received. Water that was ion-exchanged and distilled with Shimadzu SWAC-500 was used in all the preparations.

\subsection{Preparation of Materials}

2.2.1. Silica Particles. Silica particles were prepared by the solgel method at $0.2 \mathrm{M}$ TEOS, $25 \mathrm{M}$ water, and $0.01 \mathrm{M} \mathrm{NaOH}$ in a glass reactor at $35^{\circ} \mathrm{C}$ using ethanol as a solvent. Ethanol solution of the TEOS was added to a mixture of water, ethanol, and $\mathrm{NaOH}$ aqueous solution. The reaction time was $24 \mathrm{~h}$. A colloidal suspension of the silica particles was obtained, as shown in Figure 1(a). Assuming completion of reaction, the suspension had a silica concentration of $12.0 \mathrm{~g} / \mathrm{L}$. The particles were washed by repeating centrifugation, removal of supernatant, addition of water, and sonication over three times. Final volume of the suspension was adjusted to the volume of initial solution with the addition of water. Figure 1(b) shows a TEM image of the silica particles. These silica particles had an average size of $80.4 \pm 14.9 \mathrm{~nm}$.

2.2.2. Immobilization of Gd-EDTA on Silica Particles. Amino groups were first introduced on silica particle surface by using APMS, since alkoxide groups of APMS were expected to react

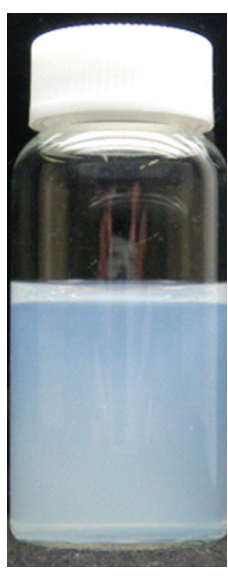

(a)

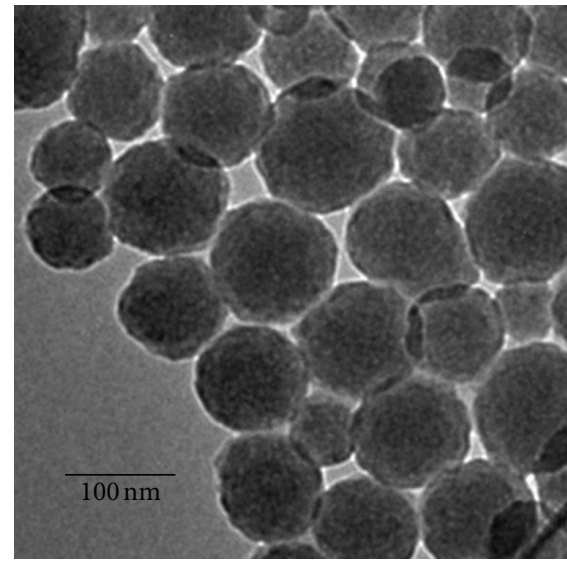

(b)
FIgURE 1: Photograph of colloid solution of silica particles and their TEM image.

with $\mathrm{OH}$ groups on silica surface of the silica particles. APMS was added to the silica particle colloid solution at $35^{\circ} \mathrm{C}$. An initial APMS concentration was $0.03 \mathrm{M}$, and the reaction time was $24 \mathrm{~h}$. The obtained $\mathrm{NH}_{2} / \mathrm{SiO}_{2}$ particle colloid solution was washed by repeating a process composed of centrifuge, removal of supernatant with decantation, addition of DMF, and shake with a vortex mixer three times. The colloid solution was concentrated with 2 -fold by reducing the amount of $\mathrm{DMF}$ at the last process.

EDTA was next immobilized on $\mathrm{NH}_{2} / \mathrm{SiO}_{2}$ particle surface through formation of amide bonds [15, 19, 20], since carboxyl groups of EDTA was expected to react with amino groups on the $\mathrm{NH}_{2} / \mathrm{SiO}_{2}$ particles. EDTA/DMF mixture was added to the $\mathrm{NH}_{2} / \mathrm{SiO}_{2}$ particle colloid solution in a volume ratio of $1: 1$ at $35^{\circ} \mathrm{C}$. The reaction time was $24 \mathrm{~h}$, and an initial EDTA concentration was $0.03 \mathrm{M}$. The obtained $\mathrm{EDTA} / \mathrm{SiO}_{2}$ particle colloid solution was washed by repeating a process composed of centrifuge, removal of supernatant with decantation, addition of water, and shake with a vortex mixer three times.

Then, $\mathrm{Gd}\left(\mathrm{NO}_{3}\right)_{3}$ aqueous solution was added to the $\mathrm{EDTA} / \mathrm{SiO}_{2}$ colloid solution, for forming Gd complex on particle surface or for producing Gd-EDTA $/ \mathrm{SiO}_{2}$ particles. An initial $\mathrm{Gd}\left(\mathrm{NO}_{3}\right)_{3}$ concentration was $0.5 \mathrm{mM}$, and the reaction temperature and time were $35^{\circ} \mathrm{C}$ and $6 \mathrm{~h}$, respectively. The obtained Gd-EDTA/ $\mathrm{SiO}_{2}$ particle colloid solution was washed by repeating a process composed of centrifuge, removal of supernatant with decantation, addition of the water, and shake with a vortex mixer three times. The asprepared Gd-EDTA/ $/ \mathrm{SiO}_{2}$ particle colloid solution was concentrated with 10 -fold by reducing the amount of water added at the last washing process (concentrated Gd-EDTA/SiO particle colloid solution).

2.3. Characterization. Morphology of the particles was investigated by transmittance electron microscopy (TEM). TEM was performed with a JEOL JEM-2000FX II microscope 
operating at $200 \mathrm{kV}$. Samples for TEM were prepared by dropping and evaporating the nanoparticle suspensions on a collodion-coated copper grid. Dozens of particle diameters in TEM images were measured to determine volume-averaged particle size and standard deviation of particle size distribution. The presence of amino groups in solutions was confirmed by performing UV-Vis spectroscopy using TNBS. A reaction between TNBS and amino group provides yellowish coloration of solution, which is attributed to absorption with an absorption peak wavelength of $347 \mathrm{~nm}$. The TNBS aqueous solution was added to the as-prepared $\mathrm{NH}_{2} / \mathrm{SiO}_{2}$ particle colloid solution, so as to make an initial TNBS concentration $0.2 \mathrm{mM}$. UV-Vis absorption spectra were measured with a Shimadzu UV-3101PC spectrometer. Introduction of amino groups and immobilization of Gd-EDTA on particle surface were qualitatively confirmed by $\zeta$-potential measurements, though the measurements possess limited quantitative usefulness in surface analysis. To measure $\zeta$ potential of particles, electrophoretic light scattering (ELS) was performed with a Brookhaven ZetaPlus zeta potential analyzer. $\mathrm{HCl}$ aqueous solution or $\mathrm{NaOH}$ aqueous solution was added to solution to vary $\mathrm{pH}$ of solution for the ELS measurement. Gd concentrations in particle colloid solutions were measured by inductively coupled plasma (ICP) emission spectroscopy. ICP measurement was performed with a Shimadzu ICPS-7510 atom emission spectrometer. Emission was detected at a wavelength of $342.247 \mathrm{~nm}$. Samples for ICP were prepared by dissolving the particles with aqua regia and then diluting the obtained solution with water. For investigation on MRI properties, $\mathrm{T}_{1}$-(longitudinal relaxation time-) weighted images of samples and $\mathrm{T}_{1}$ values were obtained with a Bruker AVANCE III 400WB magnetic resonance imaging system at a static magnetic field of $9.4 \mathrm{~T}$. Echo time and repetition time were 8.5 and $1500 \mathrm{~ms}$, respectively. The colloid solutions for the imaging were concentrated by centrifugation, removal of supernatant, addition of the water, and sonication.

\section{Results and Discussion}

\subsection{Morphology of Particles}

3.1.1. $\mathrm{NH}_{2} / \mathrm{SiO}_{2}$ Nanoparticles. Figure 2 shows photographs of $\mathrm{NH}_{2} / \mathrm{SiO}_{2}$ nanoparticle colloid solutions prepared at various $\mathrm{pHs}$ in surface modification with amino groups. Though the solutions were opaque, neither precipitation nor flocculation was observed in the solutions similarly to the silica particle colloid solution, which indicated that the procedure of surface modification with amino groups did not affect colloidal stability of the particle colloid solutions.

The supernatants of the $\mathrm{NH}_{2} / \mathrm{SiO}_{2}$ nanoparticle colloid solutions were obtained by centrifuging and decanting the colloid solutions. Figure 3 shows photographs of the supernatants. The supernatants were yellowish, which indicated that free APMS molecules or APMS molecules that were not introduced on particles were left in the supernatants. Figure 4 shows UV-Vis spectra of the supernatants. Each spectrum revealed an absorption peak at $347 \mathrm{~nm}$ that was attributed to chemical bond between amino group and TNBS. The absorption peak intensity increased with an increase in

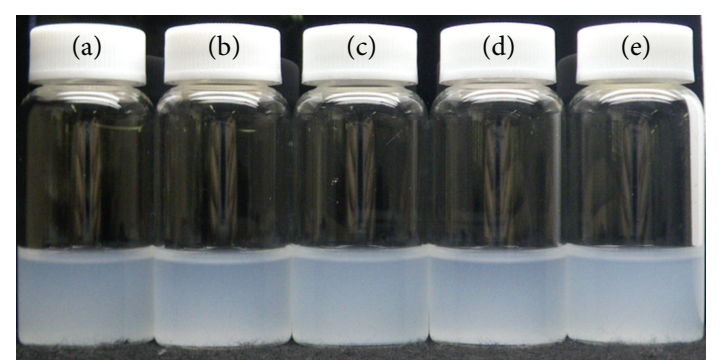

FIGURE 2: Photographs of colloid solutions of $\mathrm{NH}_{2} / \mathrm{SiO}_{2}$ particles surface modification with amino groups at pHs of (a) 3, (b) 5 , (c) 7, (d) 9, and (e) 11 .

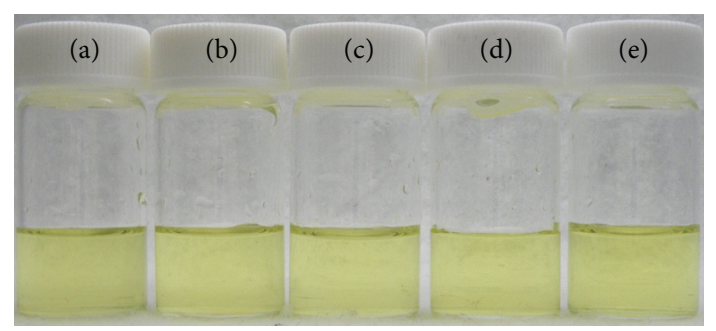

Figure 3: Photographs of supernatants of mixtures of $\mathrm{NH}_{2} / \mathrm{SiO}_{2}$ particle colloid solutions and TNBS aqueous solutions. The $\mathrm{pHs}$ in surface modification with amino groups were (a) 3, (b) 5, (c) 7, (d) 9 , and (e) 11 .

$\mathrm{pH}$, as shown in inset of Figure 4. This result meant that a large amount of APMS added were not introduced on the particle surface and remained in the supernatant at the high pHs. Surface modification with amino groups was efficient at the low pHs. A concentration of the free APMS, which should have been in the supernatant of $\mathrm{NH}_{2} / \mathrm{SiO}_{2}$ particle colloid solution, was calculated as $22.2 \mathrm{mM}$ in the final asprepared $\mathrm{NH}_{2} / \mathrm{SiO}_{2}$ particle colloid solution at $\mathrm{pH}=3$ from the absorbance of 1.30 as shown in Figure 4. A concentration of the introduced APMS was estimated at $7.8 \mathrm{mM}$ in the final $\mathrm{NH}_{2} / \mathrm{SiO}_{2}$ solution by subtracting the free APMS concentration from the initial APMS concentration of $30 \mathrm{mM}$, which meant that $6.50 \times 10^{-4}$ mole APMS was on $1 \mathrm{~g}$ silica particles.

Figure 5 shows $\zeta$-potentials as a function of $\mathrm{pH}$ for silica particles and $\mathrm{NH}_{2} / \mathrm{SiO}_{2}$ particles. $\zeta$-potentials of the silica particles were negative in a range of $\mathrm{pH}$ examined, though an absolute value of the $\zeta$-potential was lowered with decreasing $\mathrm{pH}$. The $\zeta$-potential did not become positive even at the low pHs. Such tendency of $\zeta$-potential for $\mathrm{pH}$ was typical for silica particles, as reported by other research groups [21, 22]. The $\zeta$-potential approached to zero at $\mathrm{pH}$ below 3.0, which resulted in an isoelectric point of 2-3. The isoelectric point of the $\mathrm{NH}_{2} / \mathrm{SiO}_{2}$ particles was 8.5 , which meant that the isoelectric point of silica particles shifted to high $\mathrm{pH}$ with the surface modification with amino groups. This $\mathrm{pH}$ shift was presumably derived from amino group on surface of the particles. Consequently, this result supported that the particle surface was successfully modified with APMS. 


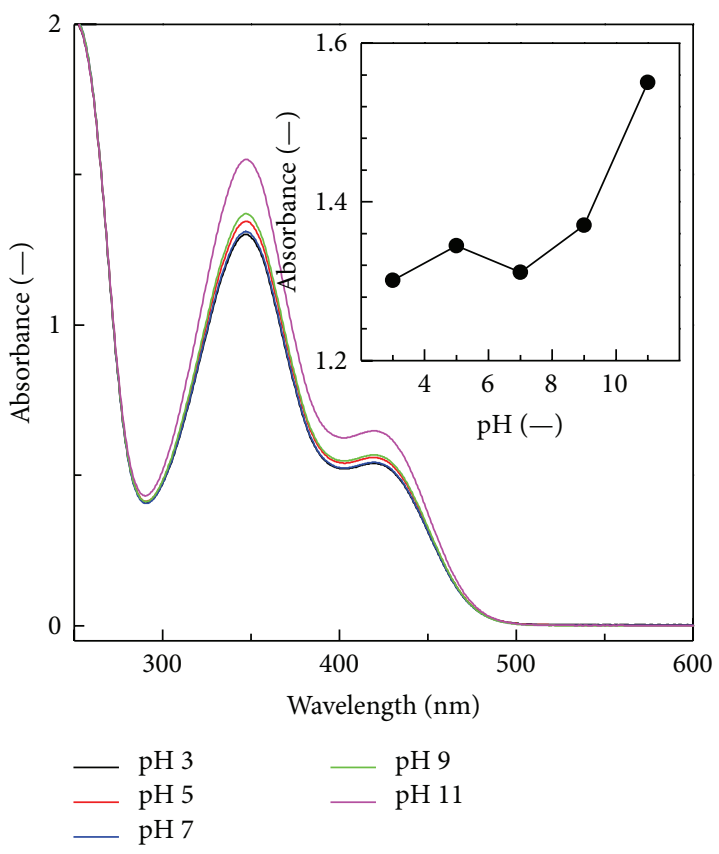

FIGURE 4: UV-Vis absorption spectra of supernatants of mixtures of $\mathrm{NH}_{2} / \mathrm{SiO}_{2}$ particle colloid solutions and TNBS aqueous solutions. The surface modification with amino groups was performed at $\mathrm{pHs}$ of $3,5,7,9$, and 11 . Inset shows absorbance at $347 \mathrm{~nm}$ as a function of $\mathrm{pH}$ of mixture of silica particle colloid solution and APMS.

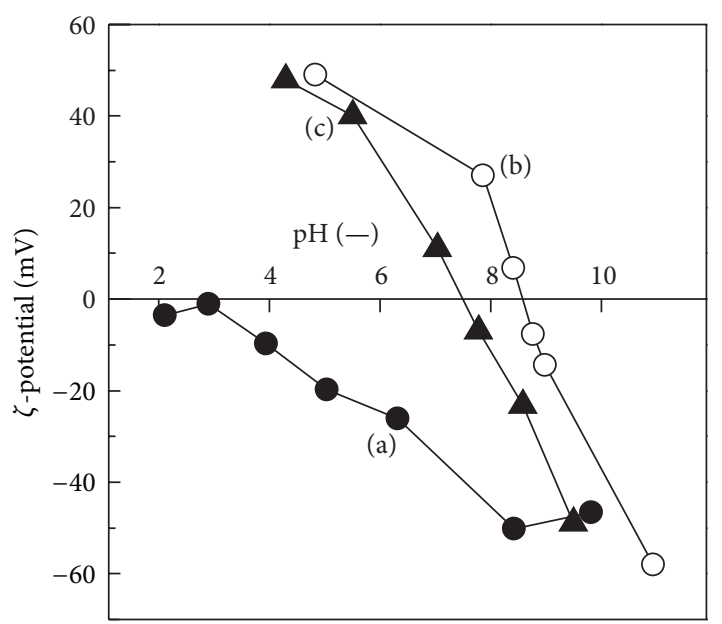

FIGURE 5: $\zeta$-potentials of (a) silica particles, (b) $\mathrm{NH}_{2} / \mathrm{SiO}_{2}$ particles, and (c) $\mathrm{Gd}-\mathrm{EDTA} / \mathrm{SiO}_{2}$ particles versus $\mathrm{pH}$.

3.1.2. Gd-EDTA/SiO $\mathrm{S}_{2}$ Nanoparticles. Figure 6(a) shows a photograph of as-prepared Gd-EDTA/SiO 2 nanoparticle colloid solution. Though the solution was opaque, neither precipitation nor flocculation was observed in the solutions similarly to the silica particle colloid solution, which indicated that the procedure of Gd-EDTA immobilization did not have an effect on colloidal stability of the particle colloid solution. Figure 6(b) shows a photograph of concentrated Gd-EDTA/ $/ \mathrm{SiO}_{2}$ nanoparticle colloid solution. Neither precipitates and nor flocculates were produced either, which indicated that the particle colloid solution was still stable colloidally even after the washing procedure.

Figure 5(c) shows $\zeta$-potential of Gd-EDTA/SiO 2 nanoparticles as a function of $\mathrm{pH}$. The isoelectric point of the Gd-EDTA $/ \mathrm{SiO}_{2}$ particles was 7.5, which meant that the isoelectric point of $\mathrm{NH}_{2} / \mathrm{SiO}_{2}$ particles shifted to low $\mathrm{pH}$ with the EDTA immobilization. Introduction of carboxyl groups derived from EDTA on particle surface would make the particle surface negative, because carboxyl groups have negative charge. Accordingly, this $\mathrm{pH}$ shift took place due to the introduction, which implied that the Gd-EDTA was immobilized on the particle surface.

ICP measurement revealed that Gd was contained in the concentrated colloid solution, which supported the implication for the successful Gd-EDTA immobilization. The Gd concentration in the concentrated colloid solution was estimated at $0.347 \mathrm{mM}$. This concentration corresponded to $0.0347 \mathrm{mM}$ Gd in the as-prepared colloid solution prior to the concentrating, which meant that $1.44 \times 10^{-6}$ mole Gd-EDTA was on $1 \mathrm{~g}$ silica particles. A Gd-EDTA-immobilization efficiency, which was defined as a ratio of ( Gd concentration in the concentrated colloid solution)/(initial Gd concentration in the as-prepared colloid solution), was estimated at $6.9 \%$, since the initial Gd concentration in the as-prepared colloid solution was $0.5 \mathrm{mM}$.

Figure 6(c) shows a TEM image of the Gd-EDTA/ $/ \mathrm{SiO}_{2}$ particles. The particles did not aggregate but were dispersed. This observation supported the high colloidal stability of the particle colloid solution revealed in Figure 6(b). The particles had an average size of $79.9 \pm 13.1 \mathrm{~nm}$.

3.2. MRI Property. Figure 7 shows $\mathrm{T}_{1}$-weighted images of the colloid solutions of Gd-EDTA/SiO 2 particles with various Gd concentrations. For $\mathrm{T}_{1}$-weighted images, strong magnetic resonance gives positive images with light contrast. All the solutions examined were clearly imaged against a black background, and the light contrast of image increased with increasing the Gd concentration.

Figure 8 shows a plot of relaxation rate $\left(1 / \mathrm{T}_{1}\right)$ of the Gd-EDTA $/ \mathrm{SiO}_{2}$ particle colloid solution as a function of the $\mathrm{Gd}$ concentration. The relaxation rate increased linearly with raising the Gd concentration. A value of relaxivity $\left(r_{1}\right)$ is defined as a slope of relaxation rate with respect to $\mathrm{Gd}$ concentration. Since active magnetic dipolar coupling occurs between protons of water molecules (solvent) and paramagnetic contrast agent, that is, active exchange of the protons with the contrast agent shortens $\mathrm{T}_{1}[23,24]$, the $r_{1}$ value is commonly used as a guideline on the performance of positive contrast agents. The $r_{1}$ value for the $\mathrm{Gd}-\mathrm{EDTA} / \mathrm{SiO}_{2}$ particle colloid solution, which was calculated by linear fitting, was $5.15 \mathrm{mM}^{-1} \cdot \mathrm{s}^{-1}$. Values of $r_{1}$ for a colloid solution of silicacoated Gd compound nanoparticles and Magnevist were 3.11 and $4.23 \mathrm{mM}^{-1} \cdot \mathrm{s}^{-1}$, respectively, according to measurements in our previous work [13]. The $r_{1}$ value of the Gd-EDTA/SiO particle colloid solution was higher than and comparable to those of silica-coated Gd compound nanoparticles and Magnevist, respectively. Since the Gd-EDTA molecules on particle surface lay close to the water protons compared 


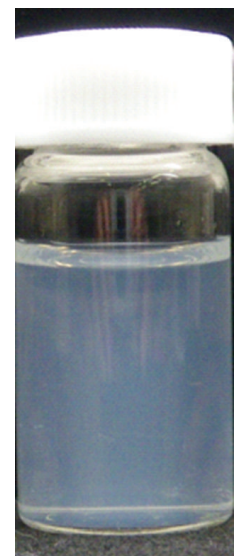

(a)

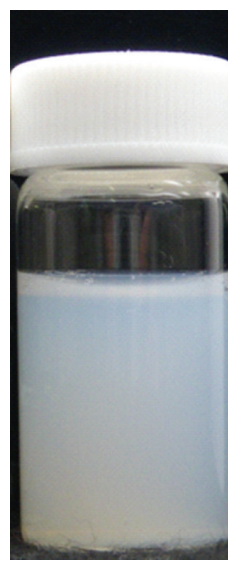

(b)

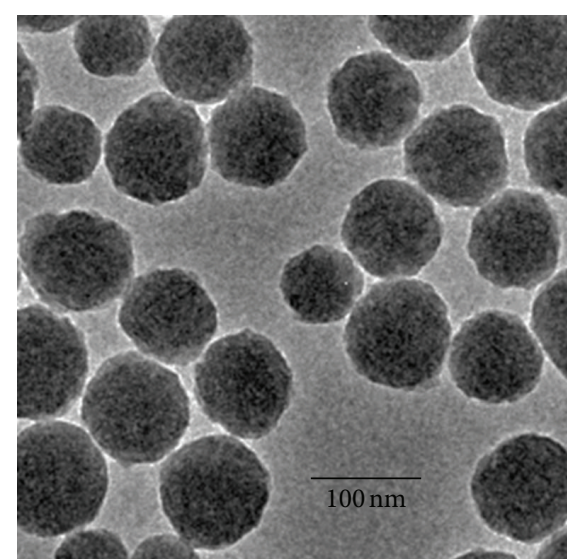

(c)

FIGURE 6: Photographs of (a) as-prepared and (b) concentrated Gd-EDTA/SiO 2 particle colloid solutions and (c) TEM image of GdEDTA/SiO 2 particles.

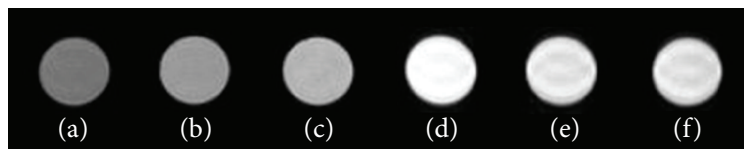

Figure 7: $\mathrm{T}_{1}$-weighted images of Gd-EDTA/ $\mathrm{SiO}_{2}$ particle colloid solutions with Gd concentrations of (a) 0, (b) 0.03, (c) 0.1, (d) 0.2, (e) 0.3 , and (f) $0.347 \mathrm{mM}$.

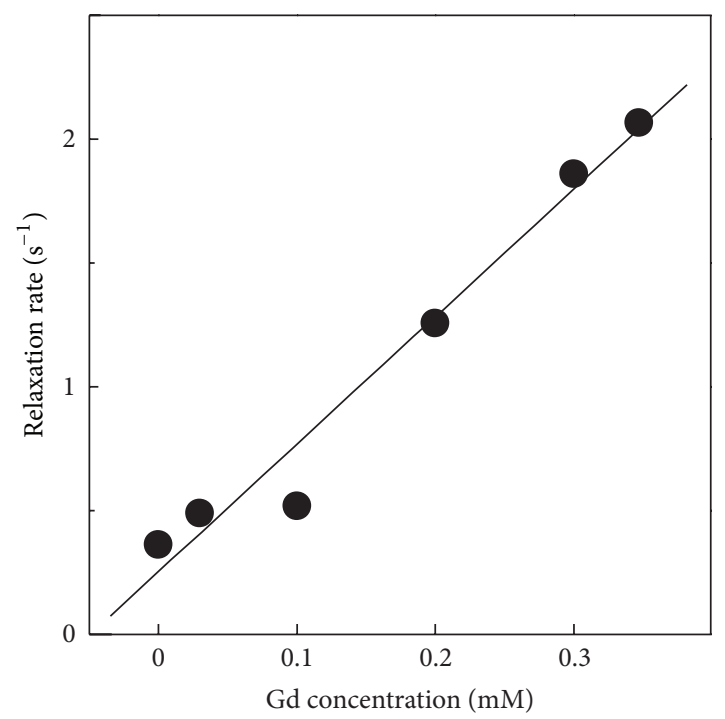

FIGURE 8: Relaxation rate $\left(1 / \mathrm{T}_{1}\right)$ of $\mathrm{Gd}-\mathrm{EDTA} / \mathrm{SiO}_{2}$ particle colloid solutions as a function of $\mathrm{Gd}$ concentration.

to the Gd compound inside the silica-coated particles, interaction between water protons and $\mathrm{Gd}$ in the Gd-EDTA/SiO particles was as significant as Magnevist.

Toxicity of silica particles [25, 26], Gd ions $[27,28]$, and chelates such as EDTA $[29,30]$ has been pointed out by several researchers. Since such materials were used for producing the Gd-EDTA/ $/ \mathrm{SiO}_{2}$ particles, it is quite required to take into account of their toxicities. Further study on toxicity of the Gd-EDTA/SiO ${ }_{2}$ particles and their colloid solutions is now on progress toward practical use of the colloid solutions as MRI contrast agents.

\section{Conclusions}

A method for producing Gd-EDTA/SiO $\mathrm{S}_{2}$ particles was proposed. The colloid solution of spherical silica particles with the size of $80.4 \pm 14.9 \mathrm{~nm}$ was prepared at $35^{\circ} \mathrm{C}$ by means of a sol-gel method using ethanol solution containing $0.2 \mathrm{M}$ TEOS, $25 \mathrm{M} \mathrm{H}_{2} \mathrm{O}$, and $0.01 \mathrm{M} \mathrm{NaOH}$. Amino groups were introduced at $\mathrm{pH} 3$ on the silica particles by using (3-aminopropyl)trimethoxysilane, and then Gd-EDTA was immobilized at pH 5 on the $\mathrm{NH}_{2} / \mathrm{SiO}_{2}$ particles. The Gd-EDTA/ $/ \mathrm{SiO}_{2}$ particles were mechanically and colloidally stable, even after concentrating the Gd$\mathrm{EDTA} / \mathrm{SiO}_{2}$ particle colloid solution up to the Gd concentration of $0.347 \mathrm{mM}$ with centrifugation. The concentrated Gd$\mathrm{EDTA} / \mathrm{SiO}_{2}$ particle colloid solution showed high-contrast $\mathrm{T}_{1}$-weighting magnetic resonance images. The relaxivity value was $5.15 \mathrm{mM}^{-1} \cdot \mathrm{s}^{-1}$, that was comparable to that for Magnevist, the commercial Gd complex contrast agent. These results obtained in the present work indicated that the Gd$\mathrm{EDTA} / \mathrm{SiO}_{2}$ particle colloid solution had an ability of MRI contrast agent.

\section{Acknowledgment}

The authors express their thanks to Professor T. Noguchi in College of Science of Ibaraki University, Japan, for his help for TEM observation.

\section{References}

[1] G. P. Yan, Z. Li, W. Xu et al., "Porphyrin-containing polyaspartamide gadolinium complexes as potential magnetic resonance imaging contrast agents," International Journal of Pharmaceutics, vol. 407, no. 1-2, pp. 119-125, 2011. 
[2] A. Kundu, H. Peterlik, M. Krssak et al., "Strategies for the covalent conjugation of a bifunctional chelating agent to albumin: synthesis and characterization of potential MRI contrast agents," Journal of Inorganic Biochemistry, vol. 105, no. 2, pp. 250-255, 2011.

[3] J. L. Gräfe, F. E. McNeill, S. H. Byun, D. R. Chettle, and M. D. Noseworthy, "The feasibility of in vivo detection of gadolinium by prompt gamma neutron activation analysis following gadolinium-based contrast-enhanced MRI," Applied Radiation and Isotopes, vol. 69, pp. 105-111, 2011.

[4] A. Makino, H. Harada, T. Okada et al., "Effective encapsulation of a new cationic gadolinium chelate into apoferritin and its evaluation as an MRI contrast agent," Nanomedicine: Nanotechnology, Biology and Medicine, vol. 7, pp. 638-646, 2011.

[5] P. Chandrasekharan, C.-X. Yong, Z. Poh et al., "Gadolinium chelate with DO3A conjugated 2-(diphenylphosphoryl)ethyldiphenylphosphonium cation as potential tumor-selective MRI contrast agent," Biomaterials, vol. 33, pp. 9225-9231, 2012.

[6] S. J. Ratnakar, N. A. Samy, and V. Alexander, "A new potential contrast agent for magnetic resonance imaging: synthesis and relaxivity studies of a gadolinium (III) complex of glucose6-phosphate conjugated 1,4,7,10-tetraazacyclododecane-1,4,7triacetic acid," Polyhedron, vol. 38, pp. 1-6, 2012.

[7] Y. Miyake, Y. Kimura, S. Ishikawa et al., "Synthesis and functional evaluation of chiral dendrimer-triamine-coordinated Gd complexes as highly sensitive MRI contrast agents," Tetrahedron Letters, vol. 53, pp. 4580-4583, 2012.

[8] S. Iwaki, K. Hanaoka, W. Piao et al., "Development of hypoxiasensitive $\mathrm{Gd}^{3+}$-based MRI contrast agents," Bioorganic \& Medicinal Chemistry Letters, vol. 22, pp. 2798-2802, 2012.

[9] A. Amiri, G. Øye, and J. Sjöblom, "Temperature and pressure effects on stability and gelation properties of silica suspensions," Colloids and Surfaces A, vol. 378, pp. 14-21, 2011.

[10] R. Mondragon, J. E. Julia, A. Barba, and J. C. Jarque, "Characterization of silica-water nanofluids dispersed with an ultrasound probe: a study of their physical properties and stability," Powder Technology, vol. 224, pp. 138-146, 2012.

[11] M. Wiśniewska, "The temperature effect on the adsorption mechanism of polyacrylamide on the silica surface and its stability," Applied Surface Science, vol. 258, pp. 3094-3101, 2012.

[12] F. Lux, A. Mignot, P. Mowat et al., "Ultrasmall rigid particles as multimodal probes for medical applications," Angewandte Chemie, vol. 50, pp. 12299-12303, 2011.

[13] Y. Kobayashi, H. Morimoto, T. Nakagawa, K. Gonda, and N. Ohuchi, "Preparation of silica-goated gadolinium compound particle colloid solution and its application in imaging," submitted to Advances in Nano Research.

[14] D. Gerion, J. Herberg, R. Bok et al., "Paramagnetic silica-coated nangcrystals as an advanced MRI contrast agent," Journal of Physical Chemistry C, vol. 111, no. 34, pp. 12542-12551, 2007.

[15] H. Yang, Y. Zhuang, Y. Sun et al., "Targeted dual-contrast $\mathrm{T}_{1}$ - and $\mathrm{T}_{2}$-weighted magnetic resonance imaging of tumors using multifunctional gadolinium-labeled superparamagnetic iron oxide nanoparticles," Biomaterials, vol. 32, no. 20, pp. 45844593, 2011.

[16] V. Feldmann, J. Engelmann, S. Gottschalk, and H. A. Mayer, "Synthesis, characterization and examination of Gd[DO3Ahexylamine]-functionalized silica nanoparticles as contrast agent for MRI-applications," Journal of Colloid and Interface Science, vol. 366, pp. 70-79, 2012.

[17] S. L. C. Pinho, H. Faneca, C. F. G. C. Geraldes, M.-H. Delville, L. D. Carlos, and J. Rocha, "Lanthanide-DTPA grafted silica nanoparticles as bimodal-imaging contrast agents," Biomaterials, vol. 33, pp. 925-935, 2012.

[18] A. Xia, M. Chen, Y. Gao, D. Wu, W. Feng, and F. Li, "Gd ${ }^{3+}$ complex-modified NaLuF4-based upconversion nanophosphors for trimodality imaging of NIR-to-NIR upconversion luminescence, X-ray computed tomography and magnetic resonance," Biomaterials, vol. 33, pp. 5394-5405, 2012.

[19] F. M. Koehler, M. Rossier, M. Waelle et al., "Magnetic EDTA: coupling heavy metal chelators to metal nanomagnets for rapid removal of cadmium, lead and copper from contaminated water," Chemical Communications, no. 32, pp. 4862-4864, 2009.

[20] K. H. Bae, Y. B. Kim, Y. Lee, J. Y. Hwang, H. Park, and T. G. Park, "Bioinspired synthesis and characterization of gadoliniumlabeled magnetite nanoparticles for dual contrast $\mathrm{T}_{1}$ - and $\mathrm{T}_{2}$ weighted magnetic resonance imaging," Bioconjugate Chemistry, vol. 21, no. 3, pp. 505-512, 2010.

[21] S. Janhom, "Polyethyleneimine/sodium dodecyl sulphate adsorbed silica particles and their adsorption properties," Colloids and Surfaces A, vol. 369, no. 1-3, pp. 186-190, 2010.

[22] E. J. Teh, Y. K. Leong, Y. Liu, B. C. Ong, C. C. Berndt, and S. B. Chen, "Yield stress and zeta potential of washed and highly spherical oxide dispersions-critical zeta potential and Hamaker constant," Powder Technology, vol. 198, no. 1, pp. 114$119,2010$.

[23] H. Bagher-Ebadian, R. Paudyal, T. N. Nagaraja, R. L. Croxen, J. D. Fenstermacher, and J. R. Ewing, "MRI estimation of gadolinium and albumin effects on water proton," NeuroImage, vol. 54, no. 1, pp. S176-S179, 2011.

[24] Y. Liu, Z. Chen, C. Liu, D. Yu, Z. Lu, and N. Zhang, "Gadolinium-loaded polymeric nanoparticles modified with Anti-VEGF as multifunctional MRI contrast agents for the diagnosis of liver cancer," Biomaterials, vol. 32, no. 22, pp. 51675176, 2011.

[25] G. Xie, J. Sun, G. Zhong, L. Shi, and D. Zhang, "Biodistribution and toxicity of intravenously administered silica nanoparticles in mice," Archives of Toxicology, vol. 84, no. 3, pp. 183-190, 2010.

[26] H. Nishimori, M. Kondoh, K. Isoda, S. I. Tsunoda, Y. Tsutsumi, and K. Yagi, "Silica nanoparticles as hepatotoxicants," European Journal of Pharmaceutics and Biopharmaceutics, vol. 72, no. 3, pp. 496-501, 2009.

[27] A. J. Gauden, P. M. Phal, and K. J. Drummond, "MRI safety; Nephrogenic systemic fibrosis and other risks," Journal of Clinical Neuroscience, vol. 17, no. 9, pp. 1097-1104, 2010.

[28] G. Marshall and C. Kasap, "Adverse events caused by MRI contrast agents: implications for radiographers who inject," Radiography, vol. 18, pp. 132-136, 2012.

[29] L. Epelde, J. Hernández-Allica, J. M. Becerril, F. Blanco, and C. Garbisu, "Effects of chelates on plants and soil microbial community: comparison of EDTA and EDDS for lead phytoextraction," Science of the Total Environment, vol. 401, no. 1-3, pp. 21-28, 2008.

[30] N. V. Ballal, M. Kundabala, S. Bhat, N. Rao, and B. S. S. Rao, "A comparative in vitro evaluation of cytotoxic effects of EDTA and maleic acid: root canal irrigants," Oral Surgery, Oral Medicine, Oral Pathology, Oral Radiology and Endodontology, vol. 108, no. 4, pp. 633-638, 2009. 

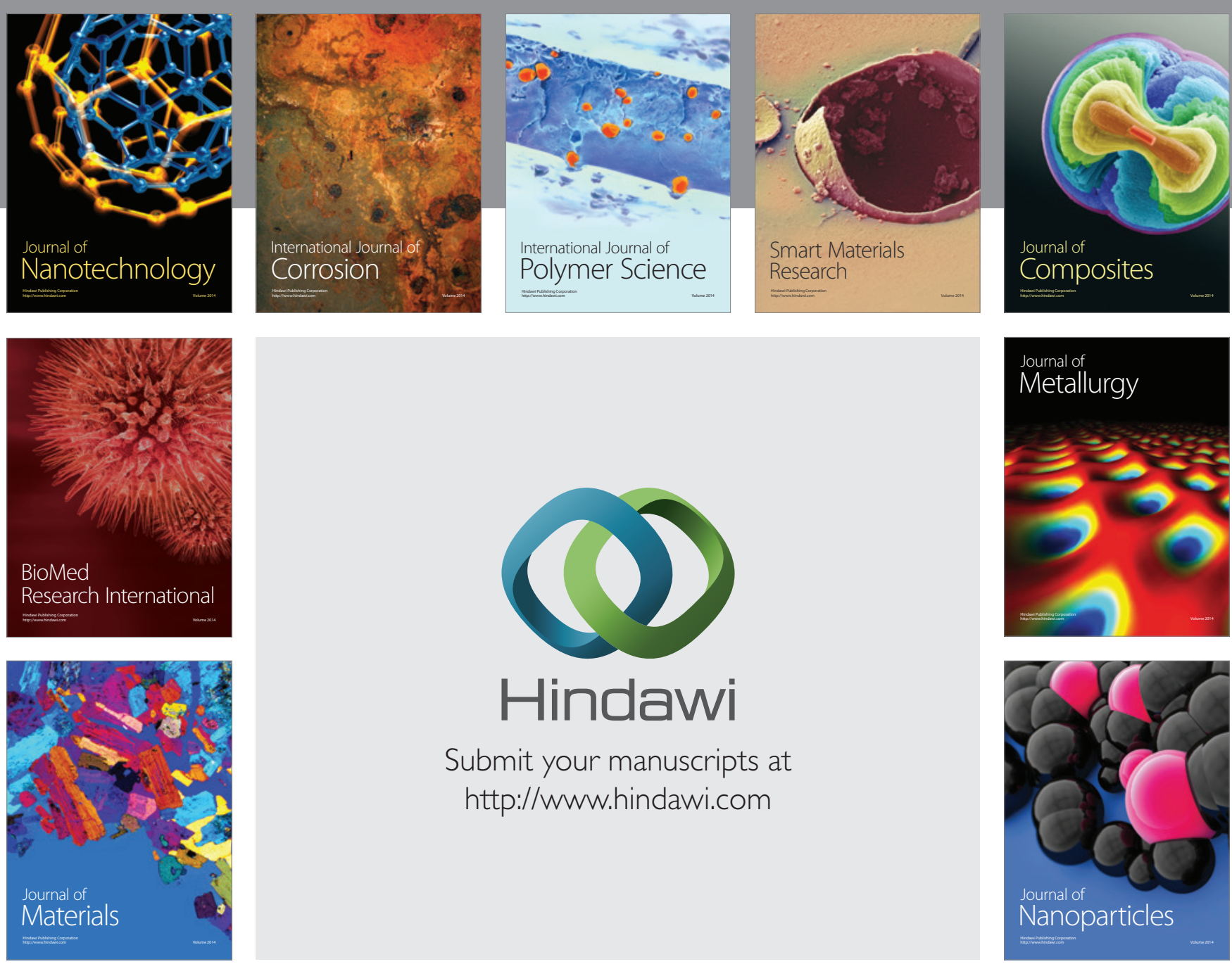

Submit your manuscripts at http://www.hindawi.com
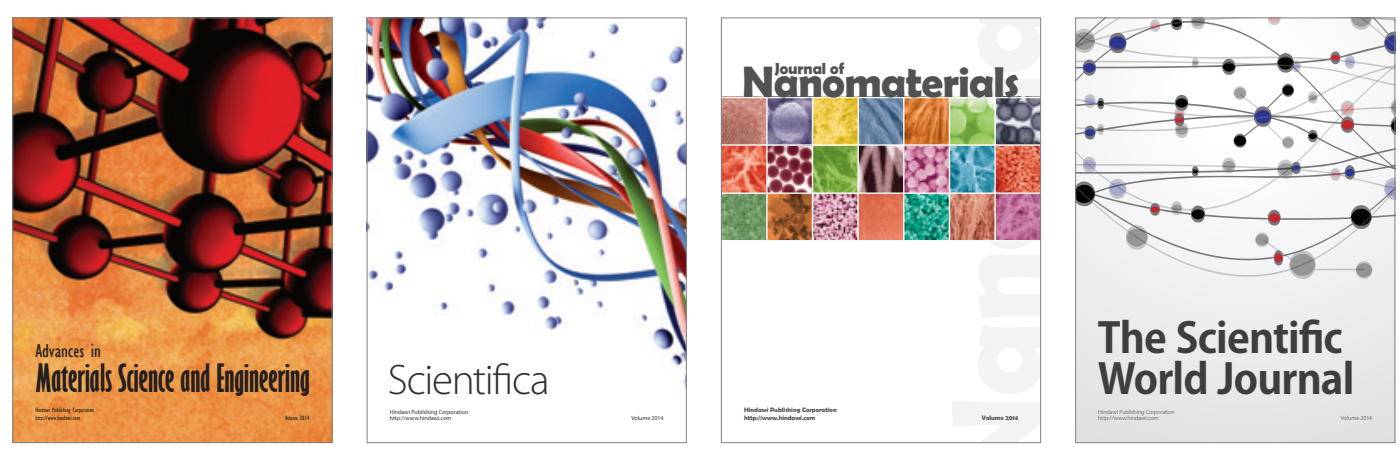

\section{The Scientific World Journal}
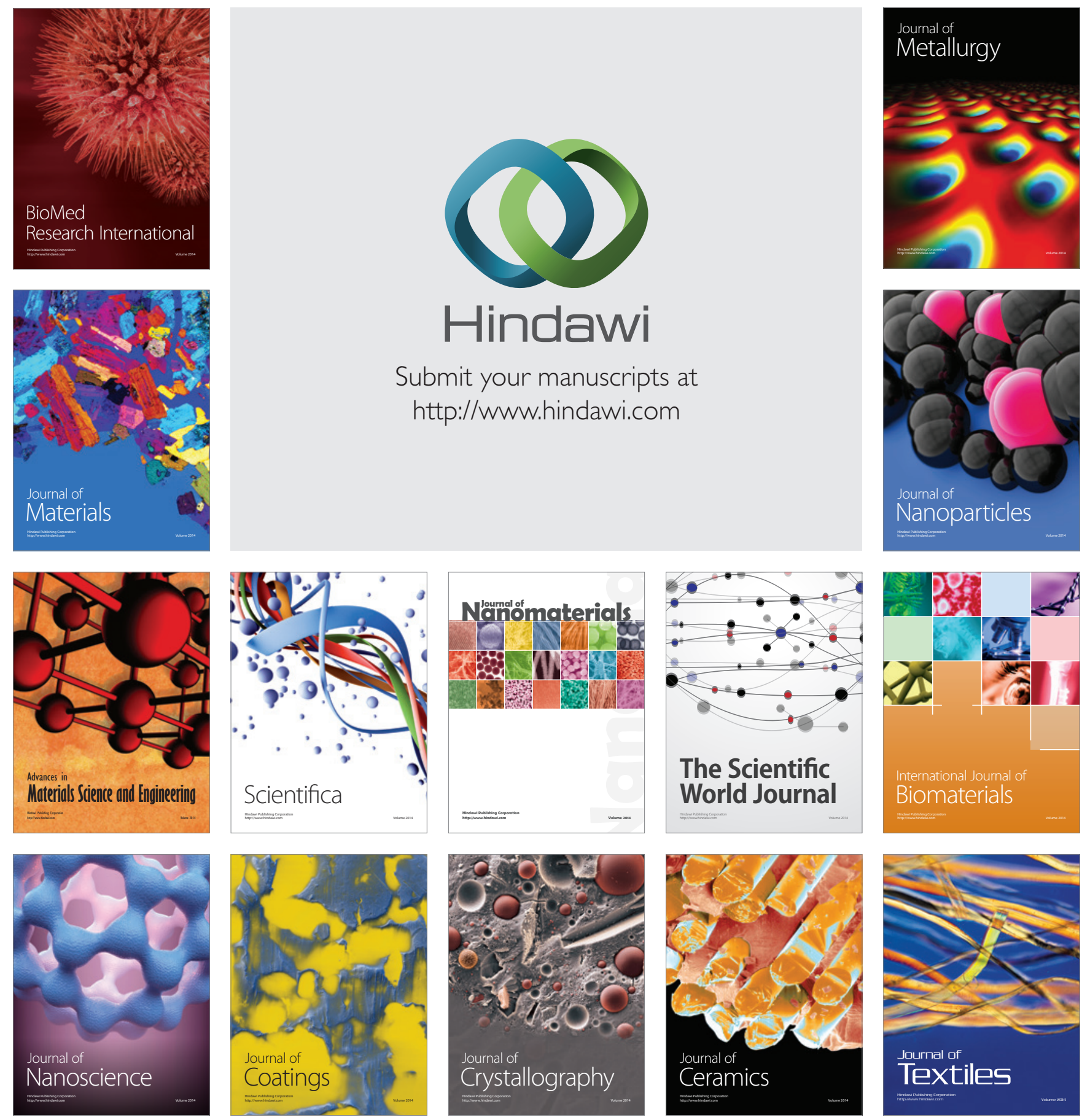